\title{
Title: Blood-based biomarkers for Alzheimer's disease: towards clinical implementation
}

Charlotte E. Teunissen, PhD, Professor. Neurochemistry Laboratory, Department of Clinical Chemistry, Amsterdam Neuroscience, Vrije Universiteit Amsterdam, Amsterdam UMC, Amsterdam, The Netherlands

Inge M.W. Verberk, PhD. Neurochemistry Laboratory, Department of Clinical Chemistry, Amsterdam Neuroscience, Vrije Universiteit Amsterdam, Amsterdam UMC, Amsterdam, The Netherlands

Elisabeth H. Thijssen, PhD. Neurochemistry Laboratory, Department of Clinical Chemistry, Amsterdam Neuroscience, Vrije Universiteit Amsterdam, Amsterdam UMC, Amsterdam, The Netherlands

Lisa Vermunt, PhD. Neurochemistry Laboratory, Department of Clinical Chemistry, Amsterdam Neuroscience, Vrije Universiteit Amsterdam, Amsterdam UMC, Amsterdam, The Netherlands

Oskar Hansson, PhD, Professor. Clinical Memory Research Unit, Department of Clinical Sciences Malmö, Lund University, Sölvegatan 18, Sweden; Memory Clinic, Skåne University Hospital, Malmö, Sweden.

Henrik Zetterberg, PhD, Professor. Institute of Neuroscience and Physiology, The Sahlgrenska Academy at the University of Gothenburg, Gothenburg, Sweden. Clinical Neurochemistry Laboratory, Sahlgrenska University Hospital, Mölndal, Sweden, UK Dementia Research Institute at UCL, London, UK, Department of Neurodegenerative Disease, UCL Institute of Neurology, London, UK. Hong Kong Center for Neurodegenerative Diseases, Hong Kong, China

Wiesje M. van der Flier, PhD, Professor. Alzheimer Center, Department of Neurology, and Department of Epidemiology and Data Science, Vije Universiteit Amsterdam, Amsterdam UMC, Amsterdam, The Netherlands.

Michelle M. Mielke, PhD, Professor. Department of Quantitative Health Sciences, and Department of Neurology, Mayo Clinic, Rochester, Minnesota, USA.

Marta Del Campo Milan, PhD. Neurochemistry Laboratory, Department of Clinical Chemistry, Amsterdam Neuroscience, Vrije Universiteit Amsterdam, Amsterdam UMC, Amsterdam, The Netherlands. Departamento de Ciencias Farmacéuticas y de la Salud, Facultad de Farmacia, Universidad San Pablo-CEU, CEU Universities, Madrid, Spain

Corresponding author:

Professor Charlotte E Teunissen

Neurochemistry Laboratory, Department of Clinical Chemistry, Amsterdam Neuroscience, Vrije Universiteit Amsterdam, Amsterdam UMC, Amsterdam, The Netherlands

De Boelelaan 1117, 1081HV

c.teunissen@amsterdamumc.nl 


\section{Abstract}

For many years, blood-based biomarkers for Alzheimer's disease seemed unattainable, but recent results have shown that they could become a reality. Convincing data generated with new highsensitivity assays have emerged with a remarkable consistency across different cohorts, but also independent of the precise analytical method used. Blood levels of amyloid and phosphorylated tau proteins associate with the corresponding cerebrospinal fluid (CSF) levels and with amyloid- or tau-PET scans. Moreover, other neurodegenerative blood-based biomarkers, such as neurofilament light and more recently glial fibrillary acidic protein, appear to provide information on disease progression and potential for monitoring treatment effects. Now the question emerges when and how we can bring these biomarkers to clinical practice. This review provides the state of the art of this highly dynamic field as described by the latest literature and guidance towards implementation for different contexts of use. 


\section{Search strategy and selection criteria}

Search of Pubmed on: 'Alzheimer's Disease' AND 'biomarker(s)' AND 'plasma' OR 'serum' OR 'blood'. Results from 2016, and older papers were included only if deemed needed to understand the subject under discussion. Papers that are published or under review or in press co-authored by the authors are also included.

Rationale: Many publications on this subject are published in the past five years and traceable on Pubmed. 


\section{Introduction}

Biomarkers that accurately reflect key aspects of Alzheimer's disease (AD) pathology during life are imperative for inclusion in clinical trials. They are also very important for an accurate diagnosis in daily clinical practice, especially now that disease-modifying therapies are becoming available. Classical pathophysiological hallmarks of $A D$ (amyloid- $\beta(A \beta)$, tau and neurodegeneration) can currently be detected using either cerebrospinal fluid (CSF) or imaging techniques, ${ }^{1}$ with amyloid- and tau-PET scans as the gold standards of amyloid and tau pathology in clinical trials. ${ }^{2}$ However, these methods are invasive and/or very expensive. Thus, there is an important medical need to identify cost-effective biomarkers that can be more easily obtained in a less invasive manner for the patient and doctor, and that can be serially measured. It is likely that blood-based biomarkers will fulfill this role.

Blood-based biomarkers for $A D$ and other dementias are now becoming a reality. Results from welldefined cohorts show high potential for implementation of the core pathological biomarkers, $A \beta$ and phosphorylated tau (pTau), ${ }^{3}$ and neurodegeneration blood markers, such as neurofilament light (NfL). Recent results show that these plasma biomarkers are abnormal in synchrony with CSF biomarker values and thus can become powerful instruments for early and precise diagnosis, prognosis or monitoring in both clinical practice and trials. The identification of blood markers is a major breakthrough in the field because it provides the option to diagnose persons with cognitive problems using a minimally invasive and cost-effective tool. Herein, we describe the state of the art of the highly dynamic and accelerating field of blood-based biomarkers for AD. We provide a comprehensive overview of recent progress focusing on three proteins that are closest to clinical implementation: the core pathological biomarkers, amyloid and pTau, and neurofilament light (NfL). We also discuss the novel emerging astrocyte biomarker glial fibrillary acidic protein (GFAP). We next discuss a blood-based biomarker roadmap towards clinical implementation, addressing the specific activities needed to enable implementation in clinical care, and in trials, highlighting the progress so far.

\section{Advances in blood-based biomarkers in sporadic AD}

\section{$A B 42$ and 40}

$A \beta$ is the main pathological hallmark of $A D$, and CSF and PET biomarkers for $A \beta$ pathology become abnormal decades before dementia symptom onset. ${ }^{1,4,5}$ A meta-analysis in 2016 showed no differences for plasma $A \beta 42$ and $A \beta 40 .{ }^{6}$ However, with the development of high-sensitive assays and technologies, recent results are much more promising. 
Different reliable methods for precise and robust quantification of plasma $A \beta 42$ and $A \beta 40$ are now available, each with its own pros and cons with regards to cost and practical aspects. Mass spectrometry (MS) assays and automated ultra-sensitive immunoassays (e.g., Simoa; panel 1) can quantify either the specific full-length or less defined $\mathrm{N}$-terminally truncated forms of these peptides (e.g., $A \beta 1-42$ and $A \beta 1-40$ or $A \beta x-42$ and $A \beta x-40) \cdot{ }^{7-21} A$ plasma test based on the mass-spectrometry analysis of $A \beta$ has been CLIA-approved to detect $A \beta$ pathology ${ }^{22}$. Other methods can detect the amyloid oligomeric tendency or Alzheimer-specific structural changes of these plasma peptides. ${ }^{23,24}$

The clinical value of the assays has been validated in different cohorts covering the complete Alzheimer's continuum and compared with the established AD biomarkers CSF A 42 and A $\beta$-PET, also in relation to cognitive performance and (risk of) cognitive decline. ${ }^{6,8}$ Plasma $A \beta 42$ to $A \beta 40$ ratio (AB42/40) identified abnormal amyloid CSF or PET status in individuals across the clinical AD continuum with accuracies ranging between $82-97 \%$ for MS assays ${ }^{8-11,22}$ and $62-79 \%$ for immunoassays. ${ }^{11-21}$ Classification performance increased with advancing disease and improved after correction for $A P O E$ $\varepsilon 4$ carriership. ${ }^{11-22}$ Of note, plasma $A \beta 42 / 40$ decreased less than $20 \%$ in individuals with cerebral $A \beta$ pathology compared with those without pathology. ${ }^{8}$ In contrast, there is about a 50\% decrease in CSF $A \beta 42 / 40$ in those with, compared to without, $A D$ pathology. ${ }^{6,8,10}$ Multiple factors may explain this difference including the production of plasma $A \beta$ from peripheral sources, binding to peripheral blood proteins that are present at $\sim 200$-fold higher concentrations than in CSF, and liver metabolic rates. In line with CSF or PET amyloid data, ${ }^{18}$ lowered plasma $A \beta 42 / 40$ or misfolded $A \beta 42$ are associated with rates of cognitive decline and risk of progression to $A D$ among cognitively normal individuals, individuals with subjective cognitive decline or individuals with mild cognitive impairment. ${ }^{13,23-29}{ }^{18}$ The diagnostic value of plasma $A \beta 42 / 40$ to differentiate $A D$ from non-AD dementias has not yet been investigated.

\section{pTau forms}

Tangles, containing hyperphosphorylated tau (pTau) in full-length or truncated forms, are the second major pathological hallmark of AD. So far, studies that measured plasma total tau found too much overlap and limited diagnostic value between clinical groups, even when using ultrasensitive technologies. ${ }^{30-34}$ This contrasts starkly with the exciting results recently obtained when analyzing the post-translational modified forms of tau. Tau has over 70 post-translational modification sites, including more than forty phosphorylation sites and several truncated forms. ${ }^{35}$ Different pTau forms are measurable in both CSF and plasma. Similar to $A \beta$, different methodologies are available, such as mass spectrometry and immune-based methods, to detect amyloid and tau pathology with high accuracy $(80-98 \%))^{7,36-44}$ 
The levels of plasma tau phosphorylated at different sites (pTau181, -217 or -231) are strongly increased (>two-fold) in clinical AD compared with both controls ${ }^{41-43,45}$ and non-AD dementias. ${ }^{37-47}$ Plasma pTau181 has gained momentum since an initial report showing increased levels in AD associated with tau-PET. ${ }^{37}$ Subsequent independent studies strongly suggest that plasma pTau181 reflects $A D$-specific neuropathology, ${ }^{38,39,48}$ as it is also elevated in $A D$ compared with non-AD dementias, including other tauopathies. ${ }^{38-40,46,48}$ Both baseline and longitudinal changes in plasma pTau181 were associated with widespread tau aggregation 6 years later. ${ }^{49}$ Moreover, pTau181 differentiated participants with amyloid pathology across different clinical stages and correlated with increased tau-PET, especially in those brain areas affected by AD pathology. ${ }^{38-40}$ In addition, increased pTau181 concentration in plasma was associated with (longitudinal) grey matter atrophy. ${ }^{38,50}$ These associations were only observed in individuals with $A \beta$ pathology ${ }^{51}$, which underpins its specificity for $A D$ neuropathological changes. pTau181 also differentiated patients with mild cognitive impairment $(\mathrm{MCl})$ who converted to $A D$ from non-converters ${ }^{39,44,52}$ and had better performance than $A \beta 42 / 40 . .^{44}$ Similar results have been observed in non-demented individuals, with high pTau181 associated with higher risk of progressing to $A D$ dementia. ${ }^{39,40} 11$

Additional promising pTau markers are emerging. Compared with pTau181, CSF pTau217 showed stronger differences between $A D$ and controls when using MS approaches, ${ }^{42,53} \mathrm{~A}$ large, multi-center cohort study showed that plasma pTau217 can differentiate AD from non-AD dementias with high accuracy (96\%), comparable to the performance of established CSF or tau-PET biomarkers. ${ }^{41}$ Plasma pTau217 also identified Tau-PET positive cases with high accuracy. Using neuropathological ratings of cerebral tau-tangle pathology, plasma pTau217 levels only correlate with the density of cortical tau pathology, and not in other tauopathies such as FTD-tau, emphasizing the specificity of plasma pTau for AD tau pathology. ${ }^{41}$ Interestingly, the study also showed that plasma pTau217 starts to increase about 20 years before onset of $\mathrm{MCl}$ in autosomal dominant $\mathrm{AD}$, which is congruent with results showing that plasma pTau217 becomes abnormal before tau-PET. ${ }^{54}$ This suggests that the origin of plasma pTau changes might not be the same as the biology causing tau-PET signal. Direct comparison studies using both MS and immunochemical platforms showed that pTau217 and pTau181 perform very well in discriminating different modalities (autopsy-confirmed AD vs FTLD, Tau- and A positivity), ${ }^{42,45,53,55,56}$. However, pTau217 could better detect A $\beta$-PET-positivity, especially when using MS approaches. ${ }^{42,53}$ pTau217 also showed slightly better accuracies when discriminating AD from other dementias and a stronger association with tau-PET imaging. ${ }^{56}$ 
A recent study analyzed plasma pTau231 in several cohorts, including a neuropathological cohort of $A D$ and non-AD dementias, and compared it to plasma pTau181. ${ }^{43}$ pTau231 differentiated AD from non-AD dementias with a performance comparable to pTau181. However, based on both A $\beta$-PET and tau Braak stages, pTau231 started to change in earlier stages of AD pathology, ${ }^{43}$ suggesting that pTau231 might be especially valuable as an early AD pathological marker even before the onset of symptoms. However, another study of a community-based population did not replicate the superiority of pTau231 over pTau181 or pTau217 for predicting amyloid or tau PET. ${ }^{57}$ Future studies should address the value of different pTau isoforms, i.e., whether the differences are truly biological or depend on the technology used.

These different tau isoforms have been shown to detect $A D$ pathology across the clinical $A D$ continuum, and may thus be used as AD-specific diagnostic markers. Evaluation in the earliest possible $A D$ stages, and in cognitively normal individuals (see section on population studies) is a crucial next step. Recently, a study in an SCD and $\mathrm{MCl}$ cohort found that the best performing model for predicting $A D$ dementia was a combination of plasma pTau(217 or 181), APOE genotype and three brief cognitive tests of memory and executive function. ${ }^{52}$ The combination of these tests could facilitate recruitment for $A D$ trials. NfL or $A \beta 42 / 40$ measured with immunoassays did not contribute to this model. ${ }^{52}$ However, another study showed that $A \beta 42 / 40$, measured by MS, outperformed both plasma pTau181 and $A \beta 42 / 40$, measured by Simoa, for detecting $A \beta-P E T$ positivity among non-demented participants. ${ }^{11}$ Additional studies are needed to determine the specificities of the tests and their most suitable context of use. In addition, unlike plasma $A \beta 42 / 40$, plasma pTau gradually increases during disease progression, ${ }^{58}$ thereby suggesting that pTau could be potentially useful to monitor disease stage. This is an exciting feature that might be useful in clinical trials of drug candidates targeted at slowing disease progression. The main limitation of the studies to date are than they have been primarily performed by a few groups utilizing retrospective cohorts in specialized centers. Prospective validation and inclusion of more heterogeneous populations are some of the next steps.

Besides phosphorylated sites, the differential truncation patterns observed in circulating tau fragments may themselves capture important aspects of AD-related neurodegeneration and cognitive decline. The N-terminal fragment of tau (NT1) in plasma is increased in AD compared to controls and predicts cognitive decline and neurodegeneration among cognitively unimpaired elderly individuals..$^{59,60}$

\section{Neurofilament light (NfL)}

$\mathrm{NfL}$ is an axonal scaffolding protein, and one of two ( $\mathrm{NfL}+\alpha-$-Internexin) core neurofilament proteins in the CNS. Neurofilaments are essential in both the growth and stability of axons, and also in synaptic 
organization and function in the CNS. ${ }^{61} \mathrm{NfL}$ has emerged as a strong cross-disease biomarker candidate for neurodegeneration. ${ }^{62,63}$ This biomarker can be measured in CSF and blood and was the first neurospecific biomarker for which clinical value was proven in a multitude of publications after development of an ultrasensitive assay.$^{64}$ Among neuroinflammatory and neurodegenerative diseases, the correlation between NfL CSF and blood levels is good to excellent ( $r$ values of 0.70 to 0.97 ). ${ }^{65}$ The highest NfL levels are seen in frontotemporal, vascular and HIV-associated dementias as well as in ALS and atypical parkinsonian disorders. ${ }^{66}$ In sporadic AD, CSF NfL shows the second highest fold-change among AD-associated fluid biomarkers (after CSF tTau). ${ }^{6}$ There is a clear association of increased CSF and plasma NfL concentrations with amyloid- and tau-PET positivity, as well as with longitudinal neurodegeneration, as determined by MRI, but with a larger overlap across groups than in familial $A D .{ }^{67,68} \mathrm{NfL}$ increases with age, which complicates interpretation of results. In clinical research and practice, $\mathrm{NfL}$ is used as a general biomarker of neuroaxonal injury or degeneration, irrespective of the underlying cause. It can be used to indicate a neurodegenerative process among patients with psychiatric symptoms. ${ }^{69}$ The biomarker could thus be used as an initial diagnostic test that, if positive, could signify additional examinations with more specific biomarkers to better understand underlying etiology. NfL could also serve as a biomarker of disease severity in clinical trials of disease-modifying treatments, and to optimize and monitor treatment effects in clinical practice, similar as for multiple sclerosis. $^{70}$

\section{Glial fibrillary acidic protein (GFAP)}

GFAP is a major cytoskeletal constituent of astrocytes. In AD, reactive astrocytosis has been implicated as a potential driver or a consequence of AD pathology. ${ }^{71}$ Both GFAP expression and protein levels were higher in areas surrounding dense $A \beta$ plaques and increased with progressing tau accumulation. ${ }^{72}$ Despite the fact that GFAP has not been as thoroughly investigated as the other proteins discussed above, promising results for its usefulness as a fluid biomarker have been recently obtained employing ultrasensitive GFAP immunoassays. Plasma or serum GFAP levels are elevated in individuals within the clinical Alzheimer's continuum. ${ }^{18,19,44,73,74}$ It differentiates abnormal A $\beta$-PET status with $81 \%$ accuracy when considering the complete clinical spectrum from cognitively unimpaired until AD dementia, ${ }^{18}$ and $76 \%$ and $80 \%$ when considering individuals without dementia only. ${ }^{18,19,75}$ Plasma GFAP levels increased linearly with increasing A $\beta$-PET burden, which disappeared at high A $\beta$-PET burden. ${ }^{76}$ Moreover, plasma/serum GFAP increases were also related to clinical disease severity, as shown by associations with syndrome diagnosis, neuropsychological test performance and MRI atrophy measures. ${ }^{18,19,73,74}$ In patients with $\mathrm{MCl}$, plasma GFAP predicted subsequent development of $\mathrm{AD}$ dementia. ${ }^{75}$ Furthermore, in cognitively unimpaired individuals, higher serum GFAP levels were 
associated with steeper rates of cognitive decline over time ${ }^{44,77,78}$ and higher risk of dementia. ${ }^{77,78}$ Of note, the predictive value of plasma or serum GFAP is independent of plasma $A \beta 42 / 40 .{ }^{18,77}$ Since astrocyte activation is not specific to Alzheimer's pathophysiology, GFAP might be a potential biomarker for other types of dementia as well. Indeed, some studies found that serum or plasma GFAP levels were increased in specific clinical and genetic subtypes of FTD. ${ }^{74,79,80}$ Levels were also elevated in Lewy body dementias compared with both controls and patients with Parkinson's disease. ${ }^{74}$ Plasma or serum GFAP could thus be used both for diagnosis (in panels) and for prognosis. However, further studies are needed to define these potential clinical applications across different types of dementia and neurodegenerative diseases. Given the early and gradual increases in GFAP co-occurring with amyloid pathology, and given its prognostic value, plasma GFAP could also play a role in trials, e.g., to support inclusion of early AD stages, enrichment, and to monitor treatment responses.

\section{Lifetime dynamics of blood-based biomarkers in genetically determined AD}

$A D$ has a monogenetic determined cause in rare cases, with a genetic mutation in the PSEN1, PSEN2, or APP genes; or a multiplication of the APP gene, which also occurs in Down syndrome due to the chromosome 21 trisomy. ${ }^{5,81}$ The advantage of studying genetically determined $A D$ is that the young age of onset (on average 40-60 years old) reduces the interference of general aging on biomarker levels. In addition, the age of onset is predictable per mutation. This allows to study biomarkers over the disease course and has contributed tremendously to the conceptual understanding of the sequence of changes in AD. ${ }^{5,81}$ Since the introduction of high-sensitivity assays, several large and small cohort studies have analyzed plasma $A \beta$, pTau, and NfL, but not yet GFAP, in genetically determined AD. $41,47,81-86$

\section{Increased plasma $A \beta 42$ in genetically determined $A D$}

Contrary to sporadic $A D$, higher plasma $A \beta 42$ and the $A \beta 42 / 40$ were detected in both autosomal dominant $A D$ mutation carriers and Down syndrome, even in children, and the levels did not correlate with their CSF counterparts. ${ }^{81,85}$ The elevated $A \beta 42$ levels in blood in genetic AD likely reflect overproduction of $A \beta 42$ caused by mutations affecting APP processing. ${ }^{87}$ However, the dynamics of plasma $A \beta 42$ levels in genetic $A D$ is not yet fully understood..$^{81,87,88} A$ recent $M S$ analysis reported a relative reduction of plasma $A \beta 42 / 40$ towards dementia onset and increased levels of $A \beta 42 / 40$ in symptomatic patients, which depended on the mutation type. ${ }^{87}$ In Down syndrome, the initially increased levels of blood $A \beta 42$ and $A \beta 42 / 40$ also decreased nearer to the time of symptom onset, followed by increased $A \beta 42$ thereafter. ${ }^{82,85}$ One should be cautious applying these results to older 
sporadic individuals (e.g., 75-80 years old). Overall, these results suggest that in genetic $A D$, plasma $A \beta$ levels are increased and fluctuate over the lifetime depending on both genetic variation and patient's disease stage, which are not yet fully understood.

Plasma pTau and NfL start to increase more than a decade before dementia onset

Biomarker dynamics of pTau and NfL in genetically determined $A D$ are consistent with sporadic $A D$ findings and provide insights in the timing of biomarker changes. Regarding pTau forms, plasma levels pTau181 and pTau217 began to increase in autosomal dominant AD 16 to 24 years before symptom onset, which aligns with the start of amyloid accumulation in the brain as measured with A $\beta$-PET and CSF A.$^{41,47,89,90}$ Like the CSF Alzheimer's biomarkers, the levels of plasma pTau appear to stabilize and may even decrease slightly after symptom onset. ${ }^{91}$ Blood NfL levels also increase early: 16 or 22 years before symptom onset in the Dominantly Inherited AD cohort study (DIAN) or in the Colombian kindred study. ${ }^{83,84}$ However, plasma NfL could distinguish between mutation carriers and unaffected family controls only 3 years before symptom onset. Similar to sporadic AD, a continued increase in NfL levels was observed in symptomatic individuals. ${ }^{83}$ Among individuals with Down syndrome, plasma $\mathrm{NfL}$ also began to increase more than 20 years before dementia onset. ${ }^{81,82}$ Furthermore, baseline and longitudinal NfL levels correlated well with other signs of neurodegeneration and clinical diagnosis, and $\mathrm{NfL}$ increases predicted future cognitive decline..$^{82,84-86,92,93}$ These findings in genetically determined AD confirm the potential use of plasma pTau181, pTau217, and NfL for distinct diagnostic, prognostic and/or disease monitoring purposes very early in the disease course.

\section{Population cohort-data and diversity perspective}

As blood-based biomarkers of amyloid and tau pathology and neurodegeneration approach clinical use, it is essential to understand what factors influence the levels of these markers to best interpret the results (figure 2). ${ }^{94}$ This information is especially important for the development of reference ranges. Because initial blood biomarker studies are conducted on well-characterized patient populations, the examination of the blood markers and the factors that affect them in diverse population- and community-based cohorts is critical. This is particularly important from a primary care standpoint, for which blood-based biomarkers are better suited compared to invasive and costly CSF and neuroimaging markers. ${ }^{95}$ However, patients in primary care have maximum heterogeneity in terms of neurodegenerative diseases and comorbidities. 
Factors such as age, sex, comorbidities, medication, lifestyle factors and genetic variation can affect the clinical interpretation of blood biomarkers. Most studies suggest that blood levels of NfL, GFAP, pTau181, pTau217, and total tau increase with age, whereas A $342 / 40$ decreases with age. ${ }^{12,58,96,97}$ This could be explained by increased prevalence of preclinical neuropathology at older ages, as well as changes in turnover of the biomarkers. Therefore, when assessing age-related associations of the ADspecific blood biomarkers, it is important to stratify by in vivo assessments of amyloid and tau pathology and to examine such associations among cognitively unimpaired individuals without pathology. The age-related aspects of non-specific markers of neurodegeneration are more difficult to study. Reports of sex differences in biomarker levels have varied. One study reported higher total tau levels for women, ${ }^{98}$ but other studies have not observed a sex difference. ${ }^{32,33,99} \mathrm{NfL}$, measured in serum or plasma, and $A \beta 42 / 40$ have not been found to differ by sex. ${ }^{11,15,96,99}$

Individuals with a higher body mass index (BMI), especially in the overweight or obese categories, have lower levels of plasma NfL. ${ }^{100}$ In a recent diverse population study, increased BMI was associated with lower levels of plasma pTau181, pTau217 and NfL, but not with levels of A $342 / A \beta 40$ or tTau. ${ }^{45}$ Although obesity is associated with brain atrophy, ${ }^{101}$ this observation is primarily explained by the higher blood volume that corresponds with increasing weight. Additional studies examining the effects of BMI on other AD-related blood-based biomarkers are needed prior to clinical implementation.

Cardiovascular comorbidities have also been shown to affect blood biomarker levels. One study reported that plasma $A \beta$ levels were lower in individuals with hypertension, ischemic heart disease and diabetes. ${ }^{12}$ However, replication in larger cohorts and with the examination of other biomarkers is needed. Renal disease can also affect blood biomarker levels because of reduced clearance.

The examination of blood-based biomarkers in diverse communities is needed to understand racial, ethnic, and geographical differences, which have been shown to influence classical AD CSF biomarker and $A \beta$-PET values. ${ }^{102,103}$ Blood biomarker studies using population diverse cohorts showed that biomarker levels were not influenced by race/ethnicity or sex. ${ }^{78}$ However, in an autopsy cohort, plasma pTau181 and 217 could better classify patients with high AD neuropathology changes in non-Hispanic black (0.94-0.96) than in non-Hispanic white (AUC:0.65-0.75)..$^{45}$ Of note, it is essential to consider differences in blood biomarker levels in the context of other factors and not to over-interpret the results. As mentioned, BMI and renal disease can affect levels of AD-related blood-based biomarkers. The age-adjusted prevalence of obesity is higher among Black and Hispanic women and men compared with white; and is higher in rural than urban areas. ${ }^{104}$ The same is true for chronic kidney disease. ${ }^{105}$ Therefore, it is important to consider racial/ethnic differences in the frequency of comorbidities and 
other factors and not simply state that there are racial/ethnic differences in an AD blood-based biomarker without any other context.

\section{The road towards clinical implementation}

Implementation of novel fluid biomarkers in clinical practice requires several aspects to be systematically addressed. The Geneva roadmap describes a five-phase framework for biomarker development. ${ }^{106}$ After initial exploratory studies (phase 1), clinical assay development and validation (phase 2) of the biomarkers are conducted. In phase 3, biomarker utility is studied detection, in retrospective and longitudinal cohorts, followed by the evaluation of the biomarkers in prospective validation studies in real-world settings (phase 4). The last phase (phase 5) is focused on implementation. ${ }^{106}$ We adapted this roadmap further with adding more technical analytical aspects of blood-based biomarkers.

To apply diagnostic assays in clinical care, assays must be approved as in vitro diagnostic assay by regulatory bodies (see Panel 2). Thus, In terms of assay requirements, phases 1-3 may employ thoroughly validated, laboratory-developed tests (LDTs) or Research-Use-Only assays (panel 2). For clinical implementation (phase 4 and 5), robust and scalable in vitro diagnostic assays approved by the certifying bodies are preferable. Another relevant prerequisite for implementation in clinical routine or trials is the development of standardized operating procedures (SOPs) for processing and storage/biobanking, preferably capable of accommodating analysis of multiple biomarkers. The influences of different pre-analytical factors ${ }^{107}$ are being carefully mapped as part of the Global Biomarker Standardization Consortium (GBSC). Such studies have already revealed that a delay in the processing of blood directly after collection or centrifugation negatively impacts $A \beta$ concentrations when kept at room temperature, irrespective of the technology used. However, this reduction can be mitigated by keeping the samples cold. No such effect was observed for pTau, NfL or GFAP, and no effects were observed for repeated freezing and thawing. The results led to a consensus standard operating procedure for blood-based biomarker collection.(personal communication CE Teunissen)

In terms of combined analyses of multiple biomarkers, validation of the biomarkers in retrospective samples (phase 3) also includes the development of diagnostic decision tools, such as algorithms employing the information provided by each of the biomarkers when assessed in panels. Since bloodbased biomarkers allow for repeated measurements, it would be relevant to understand whether the within-individual rates of change provides more information than absolute values. For example, 
change in serum $\mathrm{NfL}$ is more predictive than baseline levels among individuals with pre-symptomatic familial $A D$, while absolute levels might be more useful in the symptomatic phase. ${ }^{84}$

The evaluation of biomarkers in prospective validation studies and demonstration of clinical utility in different contexts of use is validated in phase 4 in prospective cohorts from real world settings and in individual patients (in contrast to the group level evaluations in phases 1-3). Appropriate use criteria for biofluid-based biomarkers for individual patients should be defined too (e.g., when and how they should be used). Particularly challenging is the application of biomarkers in pre-symptomatic and prodromal stages. Personalized prediction of dementia risk based on MRI and CSF biomarkers is described for $\mathrm{MCl}^{108}$ Considering that the predictive value of plasma pTau181 is similar to CSF pTau181, ${ }^{36}$ such personalized models could also be developed for blood-based biomarkers.

Communication of results to the potential users and with patients are key in phase 5 of the implementation roadmap. Even in the absence of widely available curative treatments, people highly value information, e.g., to understand the origin of their complaints, better deal with signs and symptoms, (advanced) care planning and the ability to make informed choices for the future. ${ }^{109,110}$

We indicated our estimation of the current state of development, using color codes for each of the blood-based biomarkers. Phases 1-2 have been addressed to a large extent for A , pTau ${ }^{111}$ and NfL, and work on GFAP and phase 3 is ongoing. Phases 4 and 5 still need to be addressed for all biomarkers.

We outlined the major clinical contexts of use for blood-based diagnostic biomarkers in table 1 . We expect that scenario 1 (memory clinic) might be implemented in the near future, followed by scenario 2 (diagnostic process initiated in primary care settings). Scenario 3 (population screening, often also in the primary care settings) could be envisioned in the long-term.

Currently, AD biomarker testing is very limited because of the high cost and low availability of amyloid PET and CSF biomarkers. Blood-based biomarkers may therefore enable broader biomarker testing for AD. Of note, implementation will not only depend on the outcomes of future blood-based biomarker studies, but will also differ depending on the local organization of the healthcare systems and the available treatment options. Even though the FDA has approved the anti-amyloid therapy Aducanumab, approval in other parts of the world is still awaited. Also, at this stage CSF and PET are still recommended for measurement of amyloid status, while for now blood-based biomarkers remain very promising. However, some specialized care providers might be already using blood-based biomarkers to select appropriate patients. Approval of the drug in other parts of the world, and that 
additional drug candidates are likely to follow the same route shortly, will likely further accelerate this process.

Table 1. Different scenarios of diagnostic use of blood-based biomarkers.

\begin{tabular}{|c|c|c|c|}
\hline Scenario & Description & Consequences/Impact & $\begin{array}{l}\text { Expected } \\
\text { timeframe }\end{array}$ \\
\hline 1. Memory clinic & $\begin{array}{l}\text { Added to the repertoire of } \\
\text { diagnostic tests in memory } \\
\text { clinic. Performed in addition } \\
\text { to medical/neurological } \\
\text { examination, } \\
\text { neuropsychological } \\
\text { investigation, and imaging. In } \\
\text { the future, perhaps even } \\
\text { replace CSF and/or PET for } \\
\text { AD confirmation in select } \\
\text { cases. }\end{array}$ & $\begin{array}{l}\text { Etiological diagnosis will } \\
\text { be available in all } \\
\text { memory clinics, not } \\
\text { only tertiary/ academic. } \\
\text { Broadens biomarker } \\
\text { testing for } A D \text {. }\end{array}$ & $\begin{array}{l}\text { Short term } \\
\text { (hopefully 3- } \\
5 \text { years) }\end{array}$ \\
\hline 2. Primary care provider & $\begin{array}{l}\text { To be used as a pre-screener, } \\
\text { together with a brief } \\
\text { cognitive test (e.g., MMSE or } \\
\text { MOCA). Results used to } \\
\text { reassure patients or refer for } \\
\text { further testing to memory } \\
\text { clinic. Confirmation of AD } \\
\text { pathology by CSF or PET in } \\
\text { memory clinic. }{ }^{52}\end{array}$ & $\begin{array}{l}\text { This scenario entails a } \\
\text { stepwise diagnostic } \\
\text { algorithm. Even when } \\
\text { accuracy is suboptimal, } \\
\text { this scenario is feasible. } \\
\text { Increase the number of } \\
\text { individuals detected at } \\
\text { the preclinical or } \\
\text { prodromal stage, who } \\
\text { will be followed and } \\
\text { better informed along } \\
\text { their journey directly } \\
\text { improving patient care } \\
\text { (e.g. } \\
\text { management). }\end{array}$ & $\begin{array}{l}\text { Intermediate } \\
\text { term } \\
\text { (hopefully 5- } \\
10 \text { years) }\end{array}$ \\
\hline
\end{tabular}




\begin{tabular}{|c|c|c|c|}
\hline 3. Population screening & $\begin{array}{l}\text { Three pre-requisites for } \\
\text { screening are: (i) near- } \\
\text { perfect accuracy of screening } \\
\text { test, (ii) low cost of screening } \\
\text { test, and (iii) availability of } \\
\text { treatment. Even when (i) is } \\
\text { achieved, lack of (ii) and (iii) } \\
\text { make clear that a population- } \\
\text { wide screening program for } \\
\text { AD is not yet at the horizon. }\end{array}$ & $\begin{array}{l}\text { N.A. } \\
\text { Nonetheless, we need } \\
\text { to start thinking about } \\
\text { strategies for how to } \\
\text { communicate about } \\
\text { and deal with pre- } \\
\text { symptomatic AD in the } \\
\text { community. }\end{array}$ & $\begin{array}{l}\text { Long-term } \\
\text { (Unlikely } \\
\text { within the } \\
\text { next } 10 \\
\text { years) }\end{array}$ \\
\hline
\end{tabular}

\section{Implementation of blood-based biomarkers in clinical trials}

The introduction of novel blood-based AD biomarkers is likely to improve the design and conduct of clinical trials evaluating disease-modifying therapies for $A D$. In view of the long preclinical stage of $A D,{ }^{1}$ inclusion of patients in early pre-clinical stages is, per definition, dependent on biological markers. AD blood biomarkers could be potentially used as inclusion criteria (including enrichment and stratification), and/or to evaluate target engagement and treatment efficacy (Table 2). ${ }^{112}$

The use of blood-based biomarkers to detect the presence of specific pathologies, such as amyloid plaques (and tangle formation) is very relevant for the selection of individuals eligible for treatments, such as the recently approved Aducanumab. For example, plasma pTau levels are now considered as inclusion measure in novel trials designs. Moreover, biomarkers reflecting the presence of other mechanisms, such as neuroinflammation, will be important to determine which other therapies or combination of therapies should be prescribed, which of course is also drug-dependent. Biomarker tests will likely become even more essential when disease-modifying therapies target the preclinical stages. In addition, it is also conceivable that blood-based biomarkers will become relevant to monitor biological efficacy (e.g., plasma $A \beta$ and pTau to analyze clearance of amyloid and tau aggregates) and drugs' (side-) effects such as amyloid-related imaging abnormalities (ARIA), and the safety of extending the dosing interval. ${ }^{113}$ Blood-based biomarkers are advantageous as compared to CSF and imaging biomarkers also in this context due to their non-invasiveness, lower costs, and burden to the patient and healthcare systems (e.g. tracer cost and scanning time). However, advantages of imaging include the spatial resolution and visibility of accrued damage. Lastly, Blood-based biomarkers may potentially even lower the bar in terms of cost/difficulties to conduct trials in AD.

Table 2. Different applications of blood-based biomarkers in clinical trial design. 


\begin{tabular}{|c|c|c|c|}
\hline Purpose & Markers & AD stage & Consequence \\
\hline $\begin{array}{l}\text { Prescreening in at risk } \\
\text { populations }\end{array}$ & $A \beta, p T a u$ isoforms & Predementia & $\begin{array}{l}\text { Cost-effective and } \\
\text { practical early AD } \\
\text { detection }\end{array}$ \\
\hline Inclusion criterion & $\begin{array}{l}A \beta, p T a u \text { isoforms to } \\
\text { prescreen for AD, } \\
\text { eventually combined } \\
\text { with NfL and GFAP } \\
\text { and cognitive } \\
\text { measures in an } \\
\text { algorithm yielding cut- } \\
\text { offs for a yes/no } \\
\text { decision }{ }^{114}\end{array}$ & $\begin{array}{l}\text { Predementia and } \\
\text { dementia }\end{array}$ & $\begin{array}{l}\text { Cost-effective and } \\
\text { practical early AD } \\
\text { detection. Highly } \\
\text { similar as clinical } \\
\text { diagnosis. }\end{array}$ \\
\hline $\begin{array}{l}\text { Enrichment and } \\
\text { stratification during } \\
\text { inclusion }\end{array}$ & $\begin{array}{l}\text { pTau, GFAP and NfL } \\
\text { levels, eventually split } \\
\text { into different } \\
\text { progression cut-points } \\
\text { to employ their } \\
\text { prognostic value. }\end{array}$ & $\begin{array}{l}\text { Predementia and } \\
\text { dementia }\end{array}$ & $\begin{array}{l}\text { Improves the power of } \\
\text { trials }\end{array}$ \\
\hline Target engagement & $\begin{array}{l}\text { Drug specific targets. } \\
\text { E.g., } A \beta \text { markers to } \\
\text { show targeted } A \beta \text { - } \\
\text { interfering effects. }\end{array}$ & $\begin{array}{l}\text { Predementia and } \\
\text { dementia }\end{array}$ & $\begin{array}{l}\text { Shows a biological } \\
\text { effect }\end{array}$ \\
\hline Outcome measures & $\begin{array}{l}\text { So far, only addressed } \\
\text { for NfL. }{ }^{115} \text { pTau as a } \\
\text { promising candidate. } \\
\text { Surrogacy to be } \\
\text { proven. }\end{array}$ & $\begin{array}{l}\text { Predementia and } \\
\text { dementia }\end{array}$ & $\begin{array}{l}\text { Treatment efficacy } \\
\text { and downstream } \\
\text { effects. } \\
\text { Understanding of } \\
\text { biological effects of } \\
\text { drugs. }\end{array}$ \\
\hline
\end{tabular}

\section{Outlook}


The use of blood-based biomarkers for the diagnosis and prognosis of $A D$ are nearing clinical use at both the specialty and primary care setting, largely due to the availability of ultrasensitive detection methods. A critical future step is now to define their use at the individual patient level. We expect that in a few years' time, the AD blood-based biomarkers and their assays will be ready for clinical implementation, and perhaps even earlier in clinical trials. These exciting results also hold promise for the development of novel neurospecific protein biomarkers. So far, there is a relative lack of bloodbased biomarkers reflecting the full complexity of AD pathology (figure 3), such as microglia activation or synaptic dysfunction. The difficulty with microglia biomarkers in blood is that the interference of inflammation in other organs, and no specific brain microglia biomarkers are known as yet. For synaptic dysfunction, CSF neurogranin levels have shown strong promise, but contrasting results have been obtained in blood so far. ${ }^{116}$ Nevertheless, with the emergence of feasible blood-based arrays and highthroughput proteomics technologies, novel diagnostic and prognostic biomarkers may be identified not only for AD but also for different types of dementia. Validation and implementation of blood-based biomarkers will facilitate the development of precision medicine. Importantly, the knowledge acquired from the AD biomarker field will pave the way to address the next important, unmet clinical need: identification of specific biomarkers to support the diagnosis and development of treatments for other dementia types. 
Panel 1: Technologies commonly used for blood-based biomarkers measurements.

- Sandwich enzyme-linked immunosorbent assay (ELISA): Protein concentration is measured by antibody pairs (capture and detection) able to specifically capture the analyte of interest into the wells of a plate and generate sandwich immunocomplexes (capture antibody-analyte-detection antibody). The detection antibody is labelled with an enzyme that catalyzes the conversion of a substrate to a product, which generates fluorescence or a color change proportional to the amount of analyte within the sample (usually within the nano- and pico-molar range).

- Electrochemiluminescence (ECL) immunoassays. An antibody-based approach similar to ELISA, but in this case, the detection antibody is labelled with an electrochemically active molecule that generates an electrochemiluminescence signal that is proportional to the amount of analyte within the sample. This technology is in principle more sensitive than ELISA, and involves fewer washing steps, which often result in some loss of reporter signal.

- Single molecule array (Simoa). An antibody-based approach similar to ELISA, but in this case sandwich immunocomplexes are coupled to magnetic beads rather than to a solid plate. Each single bead is loaded into its own femtoliter-sized single well with the corresponding substrate, and a fluorescence signal is then generated. The extremely low volume of the wells ( $\sim 40 \mathrm{fL}$ ) ensures a high local concentration of fluorescent signal allowing the detection of single molecules. It can thus measure proteins at very low concentrations (fM), providing 100 to 1000 times higher sensitivity than ELISA.

- Immunoprecipitation mass spectrometry (IP-MS): Antibodies coupled to beads are used to first isolate the analyte of interest from the samples. The analyte is then eluted and quantified by mass spectrometry using an isotope-labeled form of the target as internal standard.

- Immuno-infrared sensor (iRS): This technology can detect structural protein changes (e.g., protein misfolding) and has been used specifically to detect changes in the secondary structure of $A \beta$ peptides.

Panel 2: Glossary for in vitro biomarker assays

- Laboratory-developed test, or so-called 'in-house test': usually designed, developed, and used within a single laboratory. They are not legally marketed for either research or clinical use.

- 'Research-Use-Only' assay: Assays that are commercially available and approved by the regulatory authorities (e.g., European Commission [CE-marked] or the Food and Drug Administration [FDA]) 
but do not have an intended clinical decision purpose. They are not legally marketed for clinical use (e.g., Simoa or Mesoscale discovery assays).

- In vitro diagnostic assay: Assays that are intended for clinical decision-making. These are approved by the authorisation bodies (e.g., CE-marked and/or FDA-approved) and generally commercially available (e.g., Lumipulse ${ }^{\circledR} A \beta(42)+A \beta(40)$, Elecsys ${ }^{\circledR}$ Amyloid beta(42) and pTau181, C2N A $\beta(42)+$ $A \beta(40))$.

Legend Figure 1: Five-phase roadmap depicting the different phases for the development of current blood-based biomarkers for diagnosis of Alzheimer's disease.

LDTs, laboratory developed test; RUO, Research only use; SOPs, Standard Operating procedures; CSF, cerebrospinal fluid; PET, Positron emission tomography; AD, Alzheimer's disease; IVD, In vitro diagnostic. Color codes indicate the levels of evidence obtained for each marker as estimated by the authors: green: accomplished to a large extend for this marker; orange: accomplished to some extend for this marker; and red: no results are available yet that addressed this aspect for this marker

Legend Figure 2: Pathological mechanisms involved in AD and their associated body-fluid based biomarkers.

Drawing depicts the complex pathophysiology of $A D$ and highlights the body-fluid based biomarkers that can be reliably measured in both blood and CSF (colored wording) and CSF only (grey letters). Figure prepared in BioRender. 


\section{Declarations of Interests:}

Research of CET is supported by grant from the European Commission (Marie Curie International Training Network, grant agreement No 860197 (MIRIADE), and JPND), Health Holland, the Dutch Research Council (ZonMW), Alzheimer Drug Discovery Foundation, The Selfridges Group Foundation, Alzheimer Netherlands, Alzheimer Association. CET is associate editor at Alzheimer's, Research \& Therapy and Neurology, Neuroimmunology \& Neuroinflammation and Medidact.

CET has a collaboration contract with ADx Neurosciences and Quanterix, and performed contract research for AC-Immune, Axon Neurosciences, Biogen, Brainstorm Therapeutics, Celgene, EIP Pharma, Eisai, PeopleBio, Roche, Toyama, Vivoryon.

CET and WF are recipients of ABOARD, which is a public-private partnership receiving funding from ZonMW (\#73305095007) and Health Holland, Topsector Life Sciences \& Health (PPP-allowance; \#LSHM20106). More than 30 partners participate in ABOARD. ABOARD also receives funding from Edwin Bouw Fonds and Gieskes-Strijbisfonds.

IV received a research grant by Alzheimer Nederland (NL-17004).

$\mathrm{HZ}$ is a Wallenberg Scholar supported by grants from the Swedish Research Council (\#2018-02532), the European Research Council (\#681712), Swedish State Support for Clinical Research (\#ALFGBG720931), the Alzheimer Drug Discovery Foundation (ADDF), USA (\#201809-2016862), the AD Strategic Fund and the Alzheimer's Association (\#ADSF-21-831376-C, \#ADSF-21-831381-C and \#ADSF21-831377-C), the Olav Thon Foundation, the Erling-Persson Family Foundation, Stiftelsen för Gamla Tjänarinnor, Hjärnfonden, Sweden (\#FO2019-0228), the European Union's Horizon 2020 research and innovation programme under the Marie Skłodowska-Curie grant agreement No 860197 (MIRIADE), and the UK Dementia Research Institute at UCL.

$\mathrm{HZ}$ has served at scientific advisory boards for Eisai, Denali, Roche Diagnostics, Wave, Samumed, Siemens Healthineers, Pinteon Therapeutics, Nervgen, AZTherapies and CogRx, has given lectures in symposia sponsored by Cellectricon, Fujirebio, Alzecure and Biogen, and is a co-founder of Brain Biomarker Solutions in Gothenburg AB (BBS), which is a part of the GU Ventures Incubator Program. BBS has no business but owns the hybridomas for some antibodies generated in research. However, these clones are not part of any commercial products.

Research programs of WF have been funded by ZonMW, NWO, EU-FP7, EU-JPND, Alzheimer Nederland, CardioVascular Onderzoek Nederland, Health Holland, Topsector Life Sciences \& Health, Stichting Dioraphte, Gieskes-Strijbis fonds, stichting Equilibrio, Pasman stichting, Biogen MA Inc, Boehringer Ingelheim, Life-MI, AVID, Roche BV, Fujifilm, Combinostics. WF holds the Pasman chair. WF has performed contract research for Biogen MA Inc and Boehringer Ingelheim. WF has been an invited speaker at Boehringer Ingelheim, Biogen MA Inc, Eisai, Danone, WebMD Neurology (Medscape). WF is consultant to Oxford Health Policy Forum CIC, Roche and Biogen MA. WF is associate editor at Brain. All funding is paid to her institution.

$\mathrm{OH}$ : Work at the Lund University was supported by the Swedish Research Council (2016-00906), the Knut and Alice Wallenberg foundation (2017-0383), the Marianne and Marcus Wallenberg foundation (2015.0125), the Strategic Research Area MultiPark (Multidisciplinary Research in Parkinson's disease) at Lund University, the Swedish Alzheimer Foundation (AF-939932), the Swedish Brain Foundation (FO2019-0326), The Parkinson foundation of Sweden (1280/20), the Skåne University Hospital Foundation (2020-0000028), Regionalt Forskningsstöd (2020-0314) and the 
Swedish federal government under the ALF agreement (2018-Projekt0279). Doses of 18Fflutemetamol injection were sponsored by GE Healthcare.

$\mathrm{OH}$ has acquired research support (for the institution) from AVID Radiopharmaceuticals, Biogen, Eli Lilly, Eisai, GE Healthcare, Pfizer, and Roche. In the past 2 years, he has received consultancy/speaker fees from AC Immune, Alzpath, Biogen, Cerveau and Roche.

Dr Mielke has served as a consultant for Biogen and Brain Protection Company and receives research support from the United States National Institute on Aging (U01 AG06786, P30 AG62677, U54AG44170)

MC is supported by the attraction talent fellowship of Comunidad de Madrid (2018-T2/BMD-11885) and San Pablo CEU University.

The other authors declared no conflicts of interest

\section{Acknowledgement:}

The authors acknowledge the help of Sherif Bayoumi in graphical design of Figure 2.

\section{Contributions of the authors:}

$\mathrm{CT}$ : literature search, figures, writing, interpretation, overall coordination

IV: literature search, writing, interpretation, criteral review.

LT: literature search, writing, interpretation, criteral review.

LV: literature search, writing, interpretation, criteral review.

$\mathrm{OH}$ : literature search, writing, interpretation, criteral review.

$\mathrm{HZ}$ : literature search, writing, interpretation, criteral review.

WvdF: literature search, writing, interpretation, criteral review.

MM: literature search, writing, interpretation, criteral review.

MdC: literature search, writing, designing figure 2, interpretation, criteral review.

\section{References}

1. Scheltens P, De Strooper B, Kivipelto M, et al. Alzheimer's disease. Lancet 2021.

2. Hansson O. Biomarkers for neurodegenerative diseases. Nat Med 2021; 27(6): 954-63.

3. Jack CR, Jr., Bennett DA, Blennow K, et al. NIA-AA Research Framework: Toward a biological definition of Alzheimer's disease. Alzheimers Dement 2018; 14(4): 535-62.

4. Vermunt L, Sikkes SAM, van den Hout A, et al. Duration of preclinical, prodromal, and dementia stages of Alzheimer's disease in relation to age, sex, and APOE genotype. Alzheimers Dement 2019; 15(7): 888-98.

5. Bateman RJ, Xiong C, Benzinger TL, Fagan AM, Goate A, Fox NC. Clinical and biomarker changes in dominantly inherited Alzheimer's disease. N Engl J Med 2012; 367.

6. Olsson B, Lautner R, Andreasson U, et al. CSF and blood biomarkers for the diagnosis of Alzheimer's disease: a systematic review and meta-analysis. Lancet Neurol 2016; 15(7): 673-84.

7. Thijssen EH, Verberk IMW, Vanbrabant J, et al. Highly specific and ultrasensitive plasma test detects Abeta(1-42) and Abeta(1-40) in Alzheimer's disease. Sci Rep 2021; 11(1): 9736.

8. Ovod V, Ramsey KN, Mawuenyega KG, et al. Amyloid $\beta$ concentrations and stable isotope labeling kinetics of human plasma specific to central nervous system amyloidosis. Alzheimers Dement 2017; 13(8): 8419. 
9. Nakamura A, Kaneko N, Villemagne VL, et al. High performance plasma amyloid-beta biomarkers for Alzheimer's disease. Nature 2018; 554(7691): 249-54.

10. Schindler SE, Bollinger JG, Ovod V, et al. High-precision plasma beta-amyloid 42/40 predicts current and future brain amyloidosis. Neurology 2019; 93(17): e1647-e59.

11. Keshavan A, Pannee J, Karikari TK, et al. Population-based blood screening for preclinical Alzheimer's disease in a British birth cohort at age 70. Brain 2021.

12. Janelidze S, Stomrud E, Palmqvist S, et al. Plasma $\beta$-amyloid in Alzheimer's disease and vascular disease. Sci Rep 2016; 6: 26801.

13. Verberk IMW, Slot RE, Verfaillie SCJ, et al. Plasma Amyloid as Prescreener for the Earliest Alzheimer Pathological Changes. Ann Neurol 2018; 84(5): 648-58.

14. Palmqvist S, Janelidze S, Stomrud E, et al. Performance of Fully Automated Plasma Assays as Screening Tests for Alzheimer Disease-Related beta-Amyloid Status. JAMA Neurol 2019; 76(9): 1060-9.

15. Vergallo A, Mégret L, Lista S, et al. Plasma amyloid $\beta$ 40/42 ratio predicts cerebral amyloidosis in cognitively normal individuals at risk for Alzheimer's disease. Alzheimers Dement 2019; 15(6): 764-75.

16. Li WW, Shen YY, Tian DY, et al. Brain Amyloid-beta Deposition and Blood Biomarkers in Patients with Clinically Diagnosed Alzheimer's Disease. J Alzheimers Dis 2019; 69(1): 169-78.

17. De Meyer S, Schaeverbeke JM, Verberk IMW, et al. Comparison of ELISA- and SIMOA-based quantification of plasma Abeta ratios for early detection of cerebral amyloidosis. Alzheimers Res Ther 2020; 12(1): 162.

18. Verberk IMW, Thijssen E, Koelewijn J, et al. Combination of plasma amyloid beta(1-42/1-40) and glial fibrillary acidic protein strongly associates with cerebral amyloid pathology. Alzheimers Res Ther 2020; 12(1): 118.

19. Chatterjee $P$, Pedrini S, Stoops $E$, et al. Plasma glial fibrillary acidic protein is elevated in cognitively normal older adults at risk of Alzheimer's disease. Transl Psychiatry 2021; 11(1): 27.

20. de Rojas I, Romero J, Rodriguez-Gomez O, et al. Correlations between plasma and PET beta-amyloid levels in individuals with subjective cognitive decline: the Fundacio ACE Healthy Brain Initiative (FACEHBI). Alzheimers Res Ther 2018; 10(1): 119.

21. Risacher SL, Fandos N, Romero J, et al. Plasma amyloid beta levels are associated with cerebral amyloid and tau deposition. Alzheimers Dement (Amst) 2019; 11: 510-9.

22. West T, Kirmess KM, Meyer MR, et al. A blood-based diagnostic test incorporating plasma $A \beta 42 / 40$ ratio, ApoE proteotype, and age accurately identifies brain amyloid status: findings from a multi cohort validity analysis. Mol Neurodegener 2021; 16(1): 30.

23. Stockmann J, Verberk IMW, Timmesfeld N, et al. Amyloid- $\beta$ misfolding as a plasma biomarker indicates risk for future clinical Alzheimer's disease in individuals with subjective cognitive decline. Alzheimers Res Ther 2020; 12(1): 169.

24. Nabers A, Perna L, Lange J, et al. Amyloid blood biomarker detects Alzheimer's disease. EMBO Mol Med 2018; 10(5).

25. Hilal S, Wolters FJ, Verbeek MM, et al. Plasma amyloid-beta levels, cerebral atrophy and risk of dementia: a population-based study. Alzheimers Res Ther 2018; 10(1): 63.

26. Verberk IMW, Hendriksen HMA, van Harten AC, et al. Plasma amyloid is associated with the rate of cognitive decline in cognitively normal elderly: the SCIENCe project. Neurobiol Aging 2020; 89: 99-107.

27. Perez-Grijalba V, Romero J, Pesini P, et al. Plasma Abeta42/40 Ratio Detects Early Stages of Alzheimer's Disease and Correlates with CSF and Neuroimaging Biomarkers in the AB255 Study. J Prev Alzheimers Dis 2019; 6(1): 34-41.

28. Giudici KV, de Souto Barreto P, Guyonnet S, et al. Assessment of Plasma Amyloid-beta42/40 and Cognitive Decline Among Community-Dwelling Older Adults. JAMA Netw Open 2020; 3(12): e2028634.

29. Lim YY, Maruff P, Kaneko N, et al. Plasma Amyloid-beta Biomarker Associated with Cognitive Decline in Preclinical Alzheimer's Disease. J Alzheimers Dis 2020; 77(3): 1057-65.

30. Foiani MS, Woollacott IO, Heller C, et al. Plasma tau is increased in frontotemporal dementia. Journal of neurology, neurosurgery, and psychiatry 2018; 89(8): 804-7.

31. Illán-Gala I, Lleo A, Karydas A, et al. Plasma Tau and Neurofilament Light in Frontotemporal Lobar Degeneration and Alzheimer Disease. Neurology 2021; 96(5): e671-e83.

32. Mattsson N, Zetterberg H, Janelidze S, et al. Plasma tau in Alzheimer disease. Neurology 2016; 87(17): 1827-35.

33. Dage JL, Wennberg AMV, Airey DC, et al. Levels of tau protein in plasma are associated with neurodegeneration and cognitive function in a population-based elderly cohort. Alzheimers Dement 2016; 12(12): 1226-34. 
34. Zetterberg $\mathrm{H}$, Wilson D, Andreasson U, et al. Plasma tau levels in Alzheimer's disease. Alzheimers Res Ther 2013; 5(2): 9.

35. Wesseling H, Mair W, Kumar M, et al. Tau PTM Profiles Identify Patient Heterogeneity and Stages of Alzheimer's Disease. Cell 2020; 183(6): 1699-713.e13.

36. Karikari TK, Benedet AL, Ashton NJ, et al. Diagnostic performance and prediction of clinical progression of plasma phospho-tau181 in the Alzheimer's Disease Neuroimaging Initiative. Mol Psychiatry 2021; 26(2): 42942.

37. Mielke MM, Hagen CE, Xu J, et al. Plasma phospho-tau181 increases with Alzheimer's disease clinical severity and is associated with tau- and amyloid-positron emission tomography. Alzheimers Dement 2018; 14(8): 989-97.

38. Thijssen EH, La Joie R, Wolf A, et al. Diagnostic value of plasma phosphorylated tau181 in Alzheimer's disease and frontotemporal lobar degeneration. Nat Med 2020; 26(3): 387-97.

39. Janelidze S, Mattsson N, Palmqvist S, et al. Plasma P-tau181 in Alzheimer's disease: relationship to other biomarkers, differential diagnosis, neuropathology and longitudinal progression to Alzheimer's dementia. Nat Med 2020; 26(3): 379-86.

40. Karikari TK, Pascoal TA, Ashton NJ, et al. Blood phosphorylated tau 181 as a biomarker for Alzheimer's disease: a diagnostic performance and prediction modelling study using data from four prospective cohorts. Lancet Neurol 2020; 19(5): 422-33.

41. Palmqvist S, Janelidze S, Quiroz YT, et al. Discriminative Accuracy of Plasma Phospho-tau217 for Alzheimer Disease vs Other Neurodegenerative Disorders. JAMA 2020; 324(8): 772-81.

42. Barthélemy NR, Horie K, Sato C, Bateman RJ. Blood plasma phosphorylated-tau isoforms track CNS change in Alzheimer's disease. The Journal of experimental medicine 2020; 217(11).

43. Ashton NJ, Pascoal TA, Karikari TK, et al. Plasma p-tau231: a new biomarker for incipient Alzheimer's disease pathology. Acta neuropathologica 2021.

44. Simrén J, Leuzy A, Karikari TK, et al. The diagnostic and prognostic capabilities of plasma biomarkers in Alzheimer's disease. Alzheimers Dement 2021.

45. Brickman AM, Manly JJ, Honig LS, et al. Plasma p-tau181, p-tau217, and other blood-based Alzheimer's disease biomarkers in a multi-ethnic, community study. Alzheimers Dement 2021.

46. Benussi A, Karikari TK, Ashton N, et al. Diagnostic and prognostic value of serum NfL and p-Tau181 in frontotemporal lobar degeneration. Journal of neurology, neurosurgery, and psychiatry 2020; 91(9): 960-7.

47. O'Connor A, Karikari TK, Poole T, et al. Plasma phospho-tau181 in presymptomatic and symptomatic familial Alzheimer's disease: a longitudinal cohort study. Mol Psychiatry 2020.

48. Lantero Rodriguez J, Karikari TK, Suarez-Calvet M, et al. Plasma p-tau181 accurately predicts

Alzheimer's disease pathology at least 8 years prior to post-mortem and improves the clinical characterisation of cognitive decline. Acta neuropathologica 2020; 140(3): 267-78.

49. Moscoso A, Grothe MJ, Ashton NJ, et al. Time course of phosphorylated-tau181 in blood across the Alzheimer's disease spectrum. Brain 2021; 144(1): 325-39.

50. Moscoso A, Grothe MJ, Ashton NJ, et al. Longitudinal Associations of Blood Phosphorylated Tau181 and Neurofilament Light Chain With Neurodegeneration in Alzheimer Disease. JAMA Neurol 2021.

51. Tijms BM, Teunissen CE. Concatenating plasma p-tau to Alzheimer's disease. Brain 2021; 144(1): $14-7$.

52. Palmqvist S, Tideman $\mathrm{P}$, Cullen $\mathrm{N}$, et al. Prediction of future Alzheimer's disease dementia using plasma phospho-tau combined with other accessible measures. Nat Med 2021; 27(6): 1034-42.

53. Barthélemy NR, Bateman RJ, Hirtz C, et al. Cerebrospinal fluid phospho-tau T217 outperforms T181 as a biomarker for the differential diagnosis of Alzheimer's disease and PET amyloid-positive patient identification. Alzheimers Res Ther 2020; 12(1): 26.

54. Janelidze S, Berron D, Smith R, et al. Associations of Plasma Phospho-Tau217 Levels With Tau Positron Emission Tomography in Early Alzheimer Disease. JAMA Neurol 2021; 78(2): 149-56.

55. Thijssen EH, La Joie R, Strom A, et al. Plasma phosphorylated tau 217 and phosphorylated tau 181 as biomarkers in Alzheimer's disease and frontotemporal lobar degeneration: a retrospective diagnostic performance study. Lancet Neurol 2021; 20(9): 739-52.

56. Janelidze S, Stomrud E, Smith R, et al. Cerebrospinal fluid p-tau217 performs better than $\mathrm{p}$-tau181 as a biomarker of Alzheimer's disease. Nat Commun 2020; 11(1): 1683.

57. Mielke MM, Frank RD, Dage JL, et al. Comparison of Plasma Phosphorylated Tau Species With Amyloid and Tau Positron Emission Tomography, Neurodegeneration, Vascular Pathology, and Cognitive Outcomes. JAMA Neurol 2021.

58. Mattsson-Carlgren N, Janelidze S, Palmqvist S, et al. Longitudinal plasma p-tau217 is increased in early stages of Alzheimer's disease. Brain 2020; 143(11): 3234-41. 
59. Chhatwal JP, Schultz AP, Dang Y, et al. Plasma N-terminal tau fragment levels predict future cognitive decline and neurodegeneration in healthy elderly individuals. Nat Commun 2020; 11(1): 6024.

60. Chen Z, Mengel D, Keshavan A, et al. Learnings about the complexity of extracellular tau aid development of a blood-based screen for Alzheimer's disease. Alzheimers Dement 2019; 15(3): 487-96.

61. Gafson AR, Barthélemy NR, Bomont $P$, et al. Neurofilaments: neurobiological foundations for biomarker applications. Brain 2020; 143(7): 1975-98.

62. Bridel C, Verberk IMW, Heijst JJA, Killestein J, Teunissen CE. Variations in consecutive serum neurofilament light levels in healthy controls and multiple sclerosis patients. Mult Scler Relat Disord 2021; 47: 102666.

63. Khalil M, Teunissen CE, Otto M, et al. Neurofilaments as biomarkers in neurological disorders. Nat Rev Neurol 2018; 14(10): 577-89.

64. Gisslén M, Price RW, Andreasson U, et al. Plasma Concentration of the Neurofilament Light Protein (NFL) is a Biomarker of CNS Injury in HIV Infection: A Cross-Sectional Study. EBioMedicine 2016; 3: 135-40.

65. Gaetani L, Blennow K, Calabresi P, Di Filippo M, Parnetti L, Zetterberg H. Neurofilament light chain as a biomarker in neurological disorders. Journal of neurology, neurosurgery, and psychiatry 2019; 90(8): 870-81.

66. Bridel C, van Wieringen WN, Zetterberg $\mathrm{H}$, et al. Diagnostic Value of Cerebrospinal Fluid Neurofilament Light Protein in Neurology: A Systematic Review and Meta-analysis. JAMA Neurol 2019.

67. Zetterberg $\mathrm{H}$, Skillbäck T, Mattsson N, et al. Association of Cerebrospinal Fluid Neurofilament Light Concentration With Alzheimer Disease Progression. JAMA Neurol 2016; 73(1): 60-7.

68. Mattsson N, Cullen NC, Andreasson U, Zetterberg H, Blennow K. Association Between Longitudinal Plasma Neurofilament Light and Neurodegeneration in Patients With Alzheimer Disease. JAMA Neurol 2019; 76(7): 791-9.

69. Willemse EAJ, Scheltens $P$, Teunissen CE, Vijverberg EGB. A neurologist's perspective on serum neurofilament light in the memory clinic: a prospective implementation study. Alzheimers Res Ther 2021; 13(1): 101.

70. Akgün K, Kretschmann N, Haase R, et al. Profiling individual clinical responses by high-frequency serum neurofilament assessment in MS. Neurology(R) neuroimmunology \& neuroinflammation 2019; 6(3): e555.

71. Garwood CJ, Ratcliffe LE, Simpson JE, Heath PR, Ince PG, Wharton SB. Review: Astrocytes in Alzheimer's disease and other age-associated dementias: a supporting player with a central role. Neuropathol Appl Neurobiol 2017; 43(4): 281-98.

72. Simpson JE, Ince PG, Lace G, et al. Astrocyte phenotype in relation to Alzheimer-type pathology in the ageing brain. Neurobiol Aging 2010; 31(4): 578-90.

73. Elahi FM, Casaletto KB, La Joie R, et al. Plasma biomarkers of astrocytic and neuronal dysfunction in early- and late-onset Alzheimer's disease. Alzheimers Dement 2020; 16(4): 681-95.

74. Oeckl P, Halbgebauer S, Anderl-Straub S, et al. Glial Fibrillary Acidic Protein in Serum is Increased in Alzheimer's Disease and Correlates with Cognitive Impairment. J Alzheimers Dis 2019; 67(2): 481-8.

75. Cicognola C, Janelidze S, Hertze J, et al. Plasma glial fibrillary acidic protein detects Alzheimer pathology and predicts future conversion to Alzheimer dementia in patients with mild cognitive impairment. Alzheimers Res Ther 2021; 13(1): 68.

76. Asken BM, Elahi FM, La Joie R, et al. Plasma Glial Fibrillary Acidic Protein Levels Differ Along the Spectra of Amyloid Burden and Clinical Disease Stage. J Alzheimers Dis 2020; 78(1): 265-76.

77. Verberk IMW, Laarhuis MB, van den Bosch KA, et al. Serum markers glial fibrillary acidic protein and neurofilament light for prognosis and monitoring in cognitively normal older people: a prospective memory clinic-based cohort study. The Lancet Healthy Longevity 2021; 2(2): e87-e95.

78. Rajan KB, Aggarwal NT, McAninch EA, et al. Remote Blood Biomarkers of Longitudinal Cognitive Outcomes in a Population Study. Ann Neurol 2020; 88(6): 1065-76.

79. Benussi A, Ashton NJ, Karikari TK, et al. Serum Glial Fibrillary Acidic Protein (GFAP) Is a Marker of Disease Severity in Frontotemporal Lobar Degeneration. J Alzheimers Dis 2020; 77(3): 1129-41.

80. Heller C, Foiani MS, Moore K, et al. Plasma glial fibrillary acidic protein is raised in progranulinassociated frontotemporal dementia. J Neurol Neurosurg Psychiatry 2020; 91(3): 263-70.

81. Fortea J, Vilaplana E, Carmona-Iragui M, et al. Clinical and biomarker changes of Alzheimer's disease in adults with Down syndrome: a cross-sectional study. Lancet 2020; 395(10242): 1988-97.

82. Fortea J, Carmona-Iragui M, Benejam B, et al. Plasma and CSF biomarkers for the diagnosis of Alzheimer's disease in adults with Down syndrome: a cross-sectional study. Lancet Neurol 2018; 17(10): 860-9. 83. Quiroz YT, Zetterberg H, Reiman EM, et al. Plasma neurofilament light chain in the presenilin 1 E280A autosomal dominant Alzheimer's disease kindred: a cross-sectional and longitudinal cohort study. Lancet Neurol 2020; 19(6): 513-21. 
84. Preische O, Schultz SA, Apel A, et al. Serum neurofilament dynamics predicts neurodegeneration and clinical progression in presymptomatic Alzheimer's disease. Nat Med 2019; 25(2): 277-83.

85. Startin CM, Ashton NJ, Hamburg S, et al. Plasma biomarkers for amyloid, tau, and cytokines in Down syndrome and sporadic Alzheimer's disease. Alzheimers Res Ther 2019; 11(1): 26.

86. Weston PSJ, Poole T, O'Connor A, et al. Longitudinal measurement of serum neurofilament light in presymptomatic familial Alzheimer's disease. Alzheimers Res Ther 2019; 11(1): 19.

87. O'Connor A, Pannee J, Poole T, et al. Plasma amyloid- $\beta$ ratios in autosomal dominant Alzheimer's disease: the influence of genotype. Brain 2021.

88. Fagan AM, Xiong C, Jasielec MS, et al. Longitudinal change in CSF biomarkers in autosomal-dominant Alzheimer's disease. Sci Transl Med 2014; 6(226): 226 ra30.

89. Gordon BA, Blazey TM, Su Y, et al. Spatial patterns of neuroimaging biomarker change in individuals from families with autosomal dominant Alzheimer's disease: a longitudinal study. Lancet Neurol 2018; 17(3): 241-50.

90. Barthélemy NR, Li Y, Joseph-Mathurin N, et al. A soluble phosphorylated tau signature links tau, amyloid and the evolution of stages of dominantly inherited Alzheimer's disease. Nat Med 2020; 26(3): 398407.

91. Sutphen CL, McCue L, Herries EM, et al. Longitudinal decreases in multiple cerebrospinal fluid biomarkers of neuronal injury in symptomatic late onset Alzheimer's disease. Alzheimers Dement 2018; 14(7): 869-79.

92. Sanchez-Valle R, Heslegrave A, Foiani MS, et al. Serum neurofilament light levels correlate with severity measures and neurodegeneration markers in autosomal dominant Alzheimer's disease. Alzheimers Res Ther 2018; 10(1): 113.

93. Petersen ME, Rafii MS, Zhang F, et al. Plasma Total-Tau and Neurofilament Light Chain as Diagnostic Biomarkers of Alzheimer's Disease Dementia and Mild Cognitive Impairment in Adults with Down Syndrome. $J$ Alzheimers Dis 2021; 79(2): 671-81.

94. Mielke MM. Consideration of Sex Differences in the Measurement and Interpretation of Alzheimer Disease-Related Biofluid-Based Biomarkers. The journal of applied laboratory medicine 2020; 5(1): 158-69. 95. O'Bryant SE, Mielke MM, Rissman RA, et al. Blood-based biomarkers in Alzheimer disease: Current state of the science and a novel collaborative paradigm for advancing from discovery to clinic. Alzheimers Dement 2017; 13(1): 45-58.

96. Khalil M, Pirpamer L, Hofer E, et al. Serum neurofilament light levels in normal aging and their association with morphologic brain changes. Nat Commun 2020; 11(1): 812.

97. Mielke MM, Syrjanen JA, Blennow K, et al. Plasma and CSF neurofilament light: Relation to longitudinal neuroimaging and cognitive measures. Neurology 2019; 93(3): e252-e60.

98. Pase MP, Beiser AS, Himali JJ, et al. Assessment of Plasma Total Tau Level as a Predictive Biomarker for Dementia and Related Endophenotypes. JAMA Neurol 2019; 76(5): 598-606.

99. Baldacci F, Lista S, Manca ML, et al. Age and sex impact plasma NFL and t-Tau trajectories in individuals with subjective memory complaints: a 3-year follow-up study. Alzheimers Res Ther 2020; 12(1): 147.

100. Manouchehrinia A, PiehI F, Hillert J, et al. Confounding effect of blood volume and body mass index on blood neurofilament light chain levels. Ann Clin Transl Neurol 2020; 7(1): 139-43.

101. Nota MHC, Vreeken D, Wiesmann M, Aarts EO, Hazebroek EJ, Kiliaan AJ. Obesity affects brain structure and function- rescue by bariatric surgery? Neuroscience and biobehavioral reviews 2020; 108: 646-57.

102. Morris JC, Schindler SE, McCue LM, et al. Assessment of Racial Disparities in Biomarkers for Alzheimer Disease. JAMA Neurol 2019; 76(3): 264-73.

103. Gottesman RF, Schneider AL, Zhou Y, et al. The ARIC-PET amyloid imaging study: Brain amyloid differences by age, race, sex, and APOE. Neurology 2016; 87(5): 473-80.

104. Hales CM, Fryar CD, Carroll MD, Freedman DS, Aoki Y, Ogden CL. Differences in Obesity Prevalence by Demographic Characteristics and Urbanization Level Among Adults in the United States, 2013-2016. Jama 2018; 319(23): 2419-29.

105. Peralta CA, Katz R, DeBoer I, et al. Racial and ethnic differences in kidney function decline among persons without chronic kidney disease. Journal of the American Society of Nephrology : JASN 2011; 22(7): 1327-34.

106. Frisoni GB, Boccardi M, Barkhof F, et al. Strategic roadmap for an early diagnosis of Alzheimer's disease based on biomarkers. Lancet Neurol 2017; 16(8): 661-76.

107. Hansson O, Batrla R, Brix B, et al. The Alzheimer's Association international guidelines for handling of cerebrospinal fluid for routine clinical measurements of amyloid $\beta$ and tau. Alzheimers Dement 2021. 
108. van Maurik IS, Vos SJ, Bos I, et al. Biomarker-based prognosis for people with mild cognitive impairment (ABIDE): a modelling study. Lancet Neurol 2019; 18(11): 1034-44.

109. Sheffrin M, Stijacic Cenzer I, Steinman MA. Desire for predictive testing for Alzheimer's disease and impact on advance care planning: a cross-sectional study. Alzheimers Res Ther 2016; 8(1): 55.

110. Largent EA, Harkins K, van Dyck CH, Hachey S, Sankar P, Karlawish J. Cognitively unimpaired adults' reactions to disclosure of amyloid PET scan results. PLoS One 2020; 15(2): e0229137.

111. Ashton NJ, Leuzy A, Karikari TK, et al. The validation status of blood biomarkers of amyloid and phospho-tau assessed with the 5-phase development framework for AD biomarkers. European journal of nuclear medicine and molecular imaging 2021.

112. Cummings J, Feldman HH, Scheltens P. The "rights" of precision drug development for Alzheimer's disease. Alzheimers Res Ther 2019; 11(1): 76.

113. Loonstra FC, Verberk IMW, Wijburg MT, et al. Serum neurofilaments as candidate biomarkers of natalizumab associated progressive multifocal leukoencephalopathy. Ann Neurol 2019; 86(2): 322-4.

114. Cullen NC, Malarstig AN, Stomrud E, Hansson O, Mattsson-Carlgren N. Accelerated inflammatory aging in Alzheimer's disease and its relation to amyloid, tau, and cognition. Sci Rep 2021; 11(1): 1965.

115. Cullen NC, Zetterberg H, Insel PS, et al. Comparing progression biomarkers in clinical trials of early Alzheimer's disease. Ann Clin Transl Neurol 2020; 7(9): 1661-73.

116. Camporesi E, Nilsson J, Brinkmalm A, et al. Fluid Biomarkers for Synaptic Dysfunction and Loss. Biomarker insights 2020; 15: 1177271920950319. 